\title{
Identification of a Dynamic System Using Ambient Vibration Measurements
}

\author{
J.-M. Kim \\ Assistant Professor, \\ Department of Dcean Civil Engineering, \\ Yosu National Fisheries University, \\ 96-1 Dunduck-dong, \\ Yosu, Chonnam 550-250, \\ Korea \\ H. Xue \\ Senior Lecturer, \\ Department of Mechanical and \\ Production Engineering, \\ National University of Singapore, \\ 10 Kent Ridge Crescent, \\ Singapore 0511
}

This paper demonstrates how ambient vibration measurements at a limited number of locations can be effectively utilized to estimate parameters of a finite element model of a large-scale structural system involving a large number of elements. System identification using ambient vibration measurements presents a challenge requiring the use of special identification techniques, which can deal with very small magnitudes of ambient vibration contaminated by noise without the knowledge of input forces. In the present study, the modal parameters such as natural frequencies, damping ratios, and mode shapes of the structural system were estimated by means of appropriate system identification techniques including the random decrement method. Moreover, estimation of parameters such as the stiffness matrix of the finite element model from the system response measured by a limited number of sensors is another challenge. In this study, the system stiffness matrix was estimated by using the quadratic optimization involving the computed and measured modal strain energy of the system, with the aid of a sensitivity relationship between each element stiffness and the modal parameters established by the second-order inverse modal perturbation theory. The finite element models thus identified represent the actual structural system very well, as their calculated dynamic characteristics satisfactorily matched the observed ones from the ambient vibration test performed on a large-scale structural system subjected primarily to ambient wind excitations. It is noted that newly developed optical fiber accelerometers were used for this ambient vibration test. The dynamic models identified by this study will be used for design of an active mass damper system to be installed on this structure for suppressing its wind vibration.

\section{Introduction}

As a U.S.-China collaborative research project, an active mass damper system is currently under development for a 310 $\mathrm{m}$ tall TV tower recently constructed in the city of Nanjing, China. Figure 1 shows the main structure of the tower. It consists of three prestressed concrete legs placed in $120 \mathrm{deg}$ with each other, each of which has a box section with four compartments. The legs are incorporated into a space frame with seven coupling ring beams placed along the height. There is a large observation hall at the level of $185 \mathrm{~m}$ and a small observation hall at $240 \mathrm{~m}$, connected by a reinforced concrete cylinder tube structure (Cheng, 1992; Ding and Ren, 1994). The objective of the active control is to reduce the wind response of the tower, especially at the small observation hall and the antenna at the top of the tower, in order to improve the human comfort of the tourists and operators as well as to enhance the tower's communication and broadcasting quality. In May of 1995, the first author of this paper and her associates performed an ambient vibration test on this structural system, for the purpose of establishing an accurate and reliable dynamic model of the tower for the use in the design of the active vibration control system.

Contributed by the Applied Mechanics Division of THE AMERICAN SOCIETY of Mechanical ENGINEERS for publication in the ASME JourNal OF APPLIED MECHANICS .

Discussion on the paper should be addressed to the Technical Editor, Professor Lewis T. Wheeler, Department of Mechanical Engineering, University of Houston, Houston, TX 77204-4792, and will be accepted until four months after final publication of the paper itself in the ASME JOURNAL OF APpliEd MECHANICs.

Manuscript received by the ASME Applied Mechanics Division, July 7, 1997; final revision, Sept. 27, 1997. Associate Technical Editor: M. Shinozuka.
The sensors used for this test were optical fiber accelerometers recently developed by the first author and her associates. Because of their advantages such as immunity to electromagnetic fields and ease in installation, optical fiber sensors recently attracted great attention in the civil engineering community. Recent developments and applications of optical fiber sensors in civil engineering are summarized in papers by Ansari (1993) and Kersey (1994). The optical fiber accelerometer used for this field testing, however, has an unique configuration totally different from those existing optical fiber sensors summarized in these papers. Each durable sensor head consists of an oscillatory circuit and transforms a measured quantity into a frequencymodulated (FM) signal with proven reliability even over a longdistance transmission. The details of the sensing mechanism are given in (Feng, 1996) based on which a similar sensor was developed earlier. The optical fiber accelerometers were extensively tested and proven to be accurate with a sufficient dynamic range (up to $25 \mathrm{~Hz}$ ) before being used for this field measurement.

The acceleration response of the structural system measured by the optical fiber sensors under ambient conditions was used to identify the dynamic characteristics of the structural system. System identification using ambient vibration measurements presents a challenge requiring the use of special identification techniques, which can deal with very small magnitudes of ambient vibration contaminated by noise in the situation where input forces usually cannot be measured.

Studies have been conducted on identification of modal parameters including natural frequencies, modal damping ratios, and mode shapes of structural systems using ambient vibration records without input measurements (see Wang and Haldar (1994) for an extensive literature review). Mathematical for- 

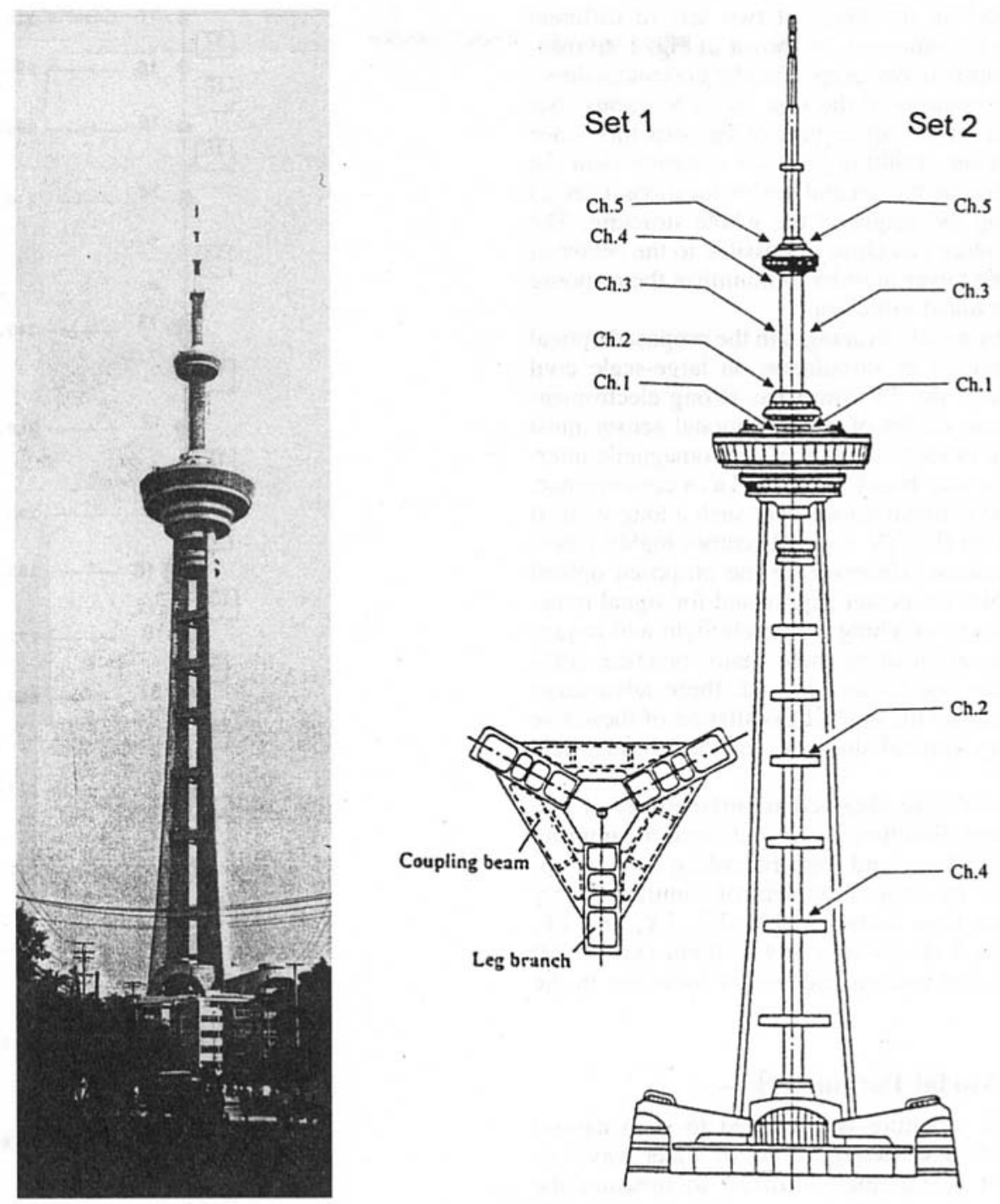

Fig. 1 Elevation, plan, and sensor locations of example structure

mulations for such a identification problem are highly complicated involving too many parameters to be identified. As a result, solutions are usually based on smooth spectral curves obtained by signal processing techniques. Among them, the random decrement technique (Cole, 1973; Ibrahim, 1977; Yang et al., 1984; Tasi and Yang, 1988) appears ideal for near-stationary wind excitations as in the case of the present field testing.

Upon identifying these model parameters, further identification of mass, stiffness, and damping matrices was carried out in order to develop the analytical model of the structural system which is important not only for design of the active mass damper controller of the structure but for evaluation of its dynamic response to wind and seismic excitations. The mass matrix in general can be estimated from the design drawings and the damping matrix constructed utilizing modal damping ratios, together with the mass and stiffness matrices. A technical challenge thus exists in the determination of the dynamic model arising from the difficulties in estimating the stiffness matrix from the ambient vibration measurements at a limited number of sensor locations.

In this study, an initial "baseline" stiffness matrix was estimated from the available design drawings of the structure. Starting from the baseline, an iterative optimization procedure was used to successively update the stiffness matrix by correlating it with the modal parameters including the natural frequencies and mode shapes measured from the field test. Available techniques for this purpose (e.g., Yun and Shinozuka, 1980; Stetson and Harrison, 1981; Tokamani and Ahmadi, 1988; Ting and Ojalvo, 1989; Hosiya and Sutoh, 1993; Okuma, 1993) usually require a sensitivity matrix of modal parameters to each element stiffness of the structural system. In this study, the second-order inverse modal perturbation theory was employed to obtain the sensitivity matrix of the natural frequencies and mode shapes to the flexural stiffness of a finite element. The first-order inverse modal perturbation technique was developed by Stetson and his colleagues for redesign problem (Stetson and Palma, 1975; Stetson and Harrison, 1981), and extended to the secondorder theory by Yun and Hong (1992) for the purpose of identifying the extent and location of structural damage. However, the problem of how to utilize the ambient vibration measurements at limited sensor locations in order to develop a stiffness matrix involving a large number of elements for a large-scale structure has not been adequately addressed in the literature, thus posing a major challenge to the present study.

\section{Ambient Vibration Measurement}

The monitoring system consisted of five optical fiber accelerometers, a single conditioner, and a tape recorder. The acceler- 
ometers were installed on the tower at two sets of different locations (with some in common), as shown in Fig. 1, to measure the ambient vibration in two perpendicular horizontal directions $(X$ and $Y$ ). The sensors at the first set of locations (Set 1) were concentrated on the upper part of the structure since this part is more flexible resulting in more vibration than the lower part, while those at the second set of locations (Set 2) were distributed along the height of the whole structure. The accelerometers were placed as close as possible to the center of the cross section of the tower in order to minimize the response component due to torsional vibration.

There are some substantial advantages in the proposed optical fiber sensor in relation to its installation on large-scale civil structures. In this study, the TV tower has strong electromagnetic fields and electric cables of a conventional sensor must be heavily shielded in order to avoid the electromagnetic interference. This results in very heavy cables and as a consequence, installation of the conventional sensor over such a long vertical distance, as required for this TV tower, becomes highly laborintensive and cumbersome. However, in the proposed optical fiber sensor, both cables for power supply and for signal transmission are optical fibers weighing extremely light and requiring no shielding, thus eliminating these major practical difficulties associated with installation. Indeed, these advantages contributed significantly to the ease of installation of these five sensors over the long vertical distances in the present field experiment.

Acceleration records were obtained simultaneously at five sensor locations in one direction each time, with a sampling time interval of $0.03125 \mathrm{sec}$ and total recording time of approximately $600 \mathrm{sec}$. In total, four sets of simultaneously measured acceleration time histories, $1 X, 1 Y, 2 X$, and $2 Y$, were recorded. Figure 3 shows two sets of them (up to 200 sec) measured at the first and second sets of locations in the $X$-direction.

\section{Estimation of Modal Parameters}

When a large-scale structure is subjected to such natural and man-made excitations as wind, traffic, or water waves or their combinations, it is extremely difficult to measure the input dynamic forces acting on the structure. As a result, only the structural response is usually measured, and frequency domain approaches (e.g., Ewins, 1984; Hong and Yun, 1992) rather than time domain approaches (e.g., Yun and Shinozuka, 1980; Shinozuka and Ghanem, 1995; Yun et al., 1997) are more appropriate for identifying modal parameters. In the present study, frequency domain identification techniques were used to extract natural frequencies, damping ratios, and mode shapes of the Nanjing TV Tower from the four sets of measured records.

3.1 Randomdec Signals. The response signal y $(t)$ measured on the structure can be decomposed into free-vibration component $\mathbf{x}_{0}(t)$ and forced-vibration component $\mathbf{x}_{f}(t)$ :

$$
\begin{aligned}
\mathbf{y}(t) & =\left\langle y_{1}(t), y_{2}(t), \ldots, y_{N_{o b}}(t)\right\rangle^{T} \\
& =\mathbf{x}_{0}(t)+\mathbf{x}_{f}(\mathbf{f}(t) ; t)
\end{aligned}
$$

where $N_{o b}$ is the number of measurement points and $\mathbf{f}(t)$ represents wind load vector which is assumed to be a random process with a zero mean. Traffic and other load can be ignored compared to the wind load because the tower structure is tall. The free-vibration component contains an impulse and/or step responses, while the forced-vibration component represents response to the random wind load. Even in the case that the complete information for the random force is not available, the modal parameters such as natural frequencies and damping ratios can be estimated accurately using signals of the free-response component. The free-vibration component can easily be



Fig. 2 Finite element model of example structure

generated by a special averaging procedure called the random decrement technique as long as measurements are carried out simultaneously at sensor locations (Ibrahim, 1977).

The randomdec signal $\mathbf{y}_{r}(\tau)$ of the measured signal is defined by Eq. (2a) and similarly corresponding force vector $\mathbf{f}_{r}(\tau)$ is defined by Eq. $(2 b)$

$$
\begin{aligned}
\mathbf{y}_{r}(\tau) & \equiv \frac{1}{N_{r}} \sum_{m=1}^{N_{r}} \mathbf{y}\left(t_{m}+\tau\right) \\
\mathbf{f}_{r}(\tau) & \equiv \frac{1}{N_{r}} \sum_{m=1}^{N_{r}} \mathbf{f}\left(t_{m}+\tau\right)
\end{aligned}
$$

where $t_{m}$ is determined from original data as all the time instants satisfying initial conditions specified for the randomdec signal, e.g., $y_{1}\left(t_{m}\right)=y_{0}$ and $\dot{y}_{1}\left(t_{m}\right) \geq 0$; and $N_{r}$ is the number of time 

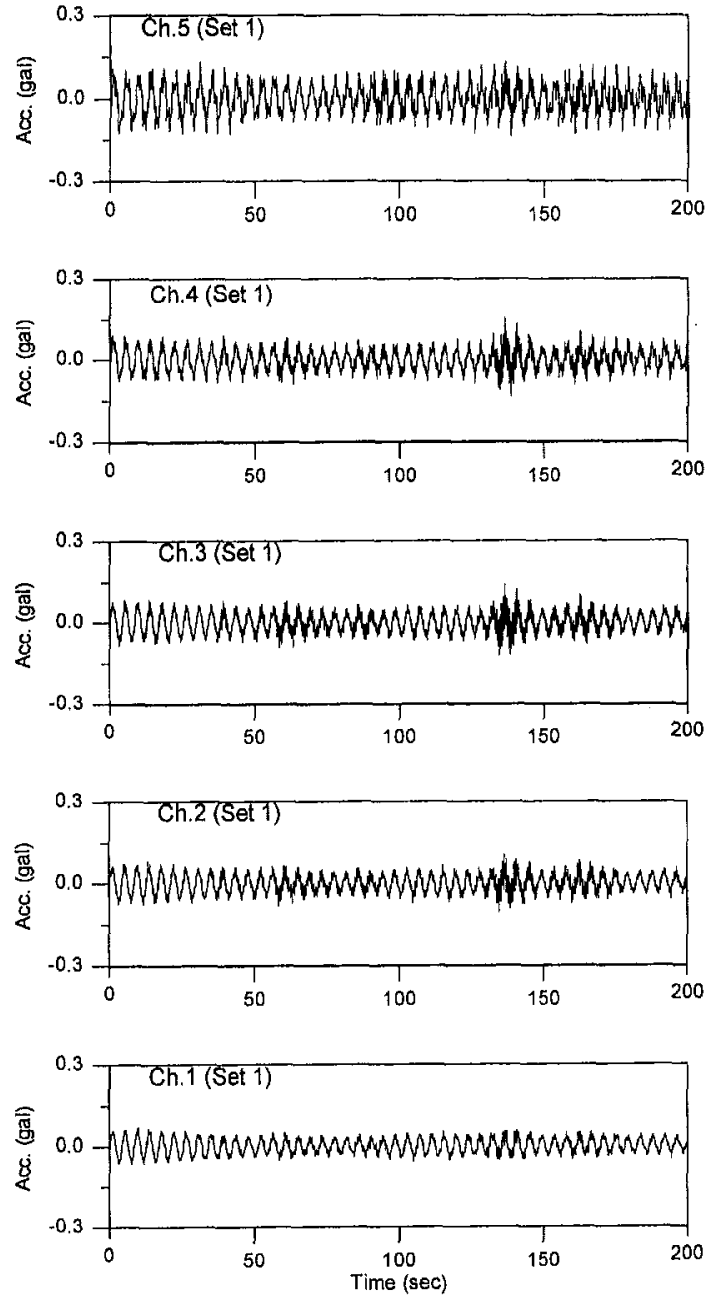

Fig. 3 Time histories of horizontal ( $X$-direction) acceleration records

points with respect to which the average is taken. If the number of data sets $N_{r}$ is sufficiently large and $\mathbf{f}(t)$ is a weakly stationary random process, the randomdec force vector $\mathbf{f}_{r}(\tau)$ will be averaged out owing to the zero-mean property of $\mathbf{f}(t)$. Thus the randomdec signal is expected to be free from the forcedvibration component under those conditions.

$$
\lim _{N_{r} \rightarrow \infty} \mathbf{f}_{r}(\tau)=\mathbf{0}
$$

In this study, $t_{m}$ 's are taken as those of zero crossing time with positive slope on the acceleration time history recorded at the small observation hall ( $\mathrm{Ch} .5$ sensor location). The total length of the randomdec signal is 4096 points. Based on these conditions, four sets of randomdec signals were obtained from the original data sets of $1 X, 2 X, 1 Y$, and $2 Y$. Figure 4 shows the randomdec time histories, and Fig. 5 displays Fourier amplitudes of typical randomdec signals compared with those of the measured signals which have been averaged using the conventional approach with data length of 8192 points and 30 percent overlap. Apparently, the time histories of the randomdec signals look like free-vibration responses, and their FFT plots are much smoother than those of the original. Now, various modal parameter estimation techniques using free-vibration measurements can be employed. It is more accurate to identify the natural frequencies and damping ratios from the randomdec signals, than from the original signals.
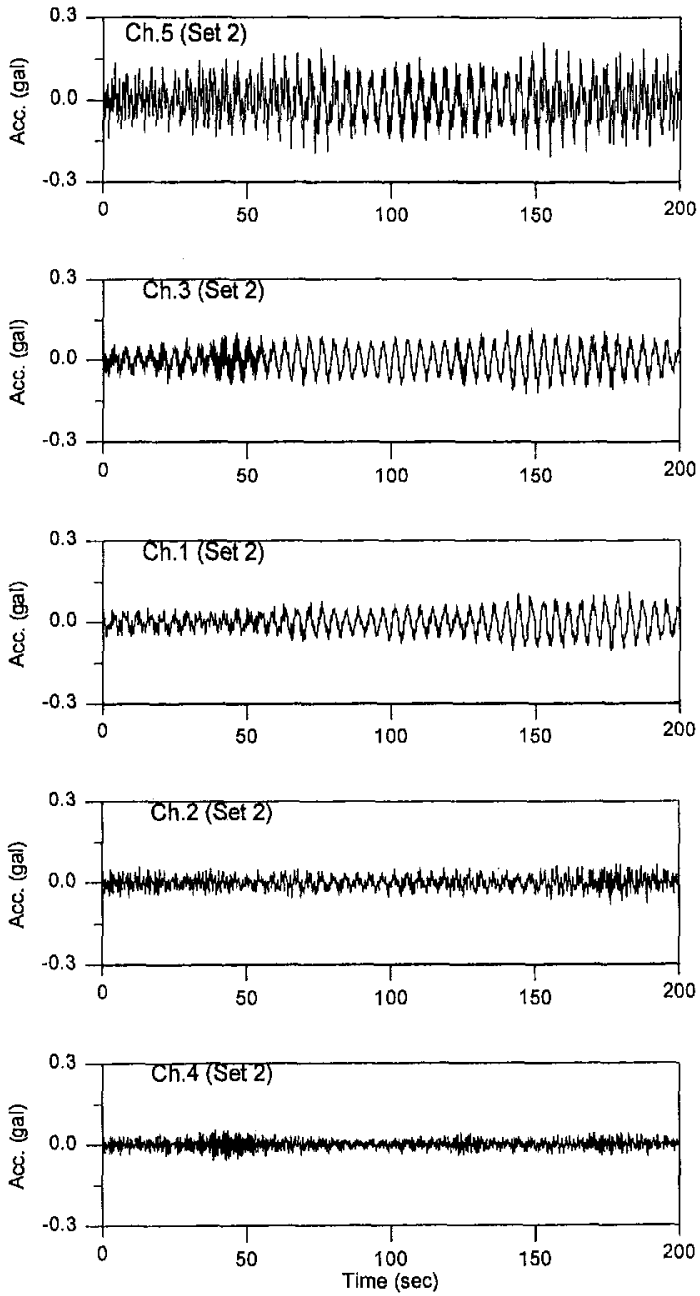

3.2 Natural Frequencies. The natural frequencies of the structural system were estimated by reading directly the frequencies at the peaks of the FFT plots of the measured acceleration averaged using the conventional approach with the total data length of 8192 points and 30 percent overlap. Because the observed frequencies might result from the dominant frequencies of the input excitations as well as structural resonance frequencies, the direct estimates were verified by checking coherence functions between two response records measured at different locations. Meanwhile, the natural frequencies were also estimated by applying the circle-fit method on the randomdec signals taking advantage of circle characteristics of frequency response function around natural frequencies in the complex plane (Ewins, 1984). The first seven natural frequencies of the structure were estimated averaging the results from the two aforementioned methods. The results are shown in Table 1 together with the estimates from the previous measurement using conventional servo-type accelerometers performed by Tang et al. (1995). It is demonstrated that the estimated frequencies based on this measurement using optical fiber accelerometers are very close to those using the conventional accelerometers. The first five identified natural frequencies were $0.237 \mathrm{~Hz}, 0.727 \mathrm{~Hz}, 1.271$ $\mathrm{Hz}, 1.588 \mathrm{~Hz}$, and $2.721 \mathrm{~Hz}$, and they are judged to be accurate since the deviation of the estimates obtained by different methods and from different data sets was very small.

The small observation hall (Ch. 5 sensor location) is an important location whose vibration is the major control target 

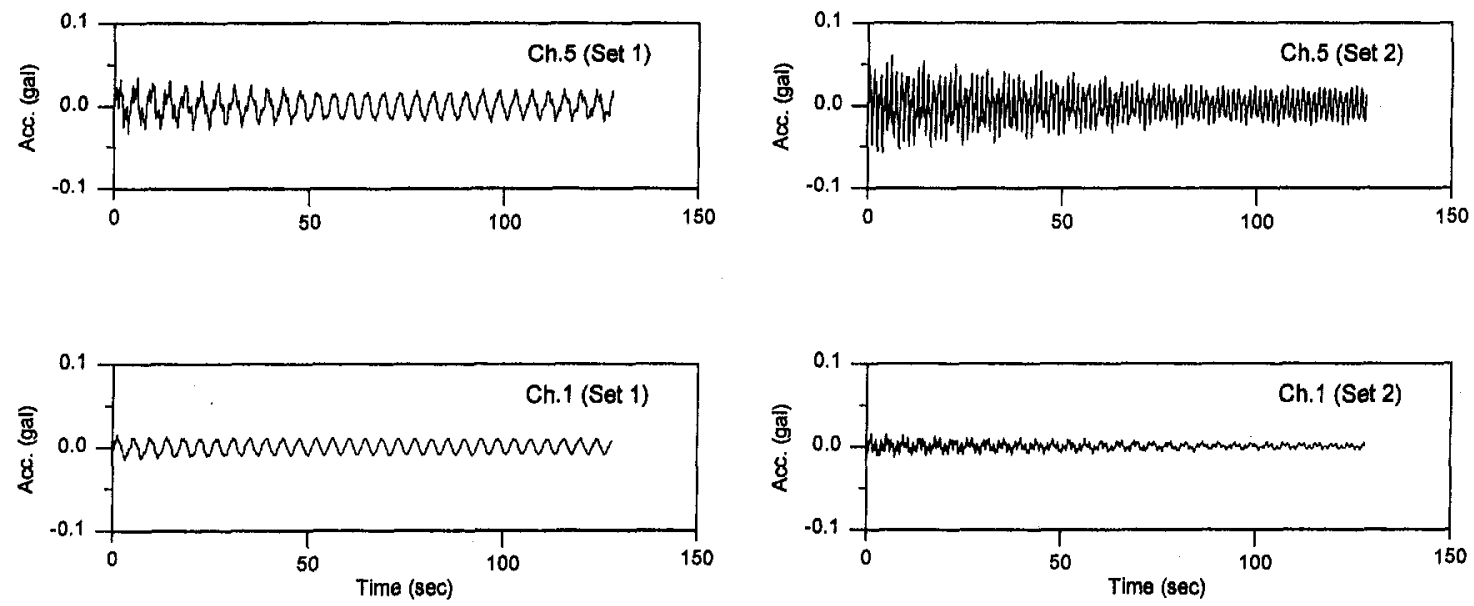

Fig. 4 Typical time histories of randomdec acceleration signals

of the active mass damper system to be installed. As seen in Fig. 6, the dominant modes associated with the response of the small observation hall are the first, second, and fourth modes. Therefore, the analytical model of the structural system to be developed from this study should well match the measured modal properties especially at these dominant modes.

A mode with a frequency around $2.5 \mathrm{~Hz}$ was identified from the acceleration recorded at the locations below the large observation hall. It appears to be a torsional mode since the sensors at those locations were placed at a distance from the vertical axis of the structure. In this study, this mode was neglected in development of a two-dimensional dynamic model for the structural system.

3.3 Damping Ratios. In general, the damping values estimated from the wind-induced structural response contains aerodynamic effects. For this reason, the direct estimation of damping ratios using the original acceleration records may result in an overestimation, and the randomdec signals free from the wind loads are ideal to use. On the other hand, the Fourier transformed response functions obtained from the randomdec signals are generally much smoother than those using the original records, as shown in Fig. 5. Therefore, damping
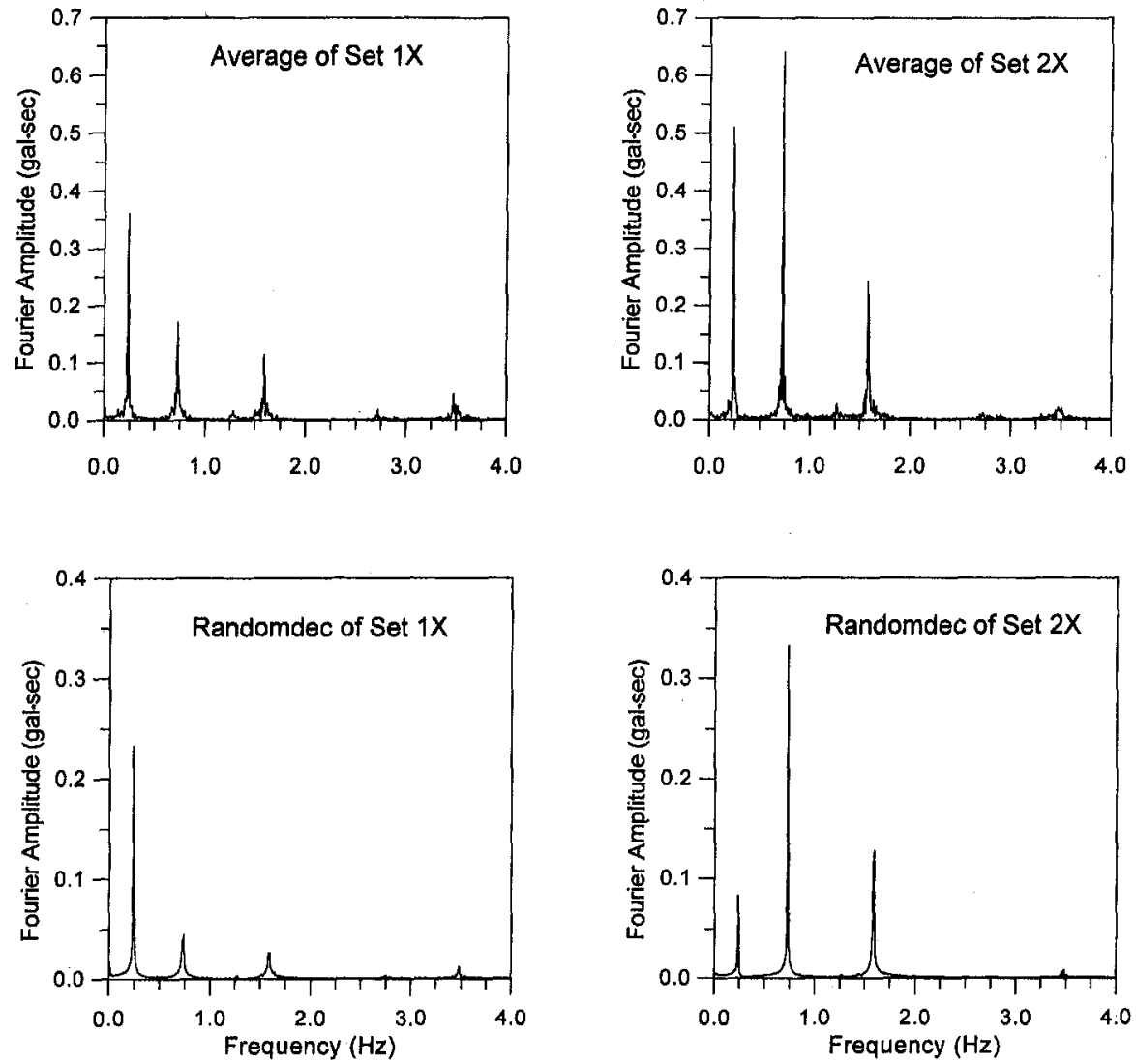

Fig. 5 Fourier amplitudes of averaged and randomdec accelerations at small hall (Ch. 5) 
Table 1 Natural frequencies of Nanjing TV Tower estimated using measured vibration data (unit: HZ)

\begin{tabular}{|c|c|c|c|c|c|c|c|}
\hline \multirow{2}{*}{$\begin{array}{l}\text { Mode } \\
\text { Number }\end{array}$} & \multicolumn{6}{|c|}{ This Study } & \multirow{2}{*}{$\begin{array}{c}\text { Tang } \\
\text { (1995) }\end{array}$} \\
\hline & $\begin{array}{c}\text { Signal } \\
\text { Used }\end{array}$ & $1 X$ & $1 Y$ & $2 X$ & $2 Y$ & Average & \\
\hline \multirow{2}{*}{1} & Original & 0.236 & 0.238 & 0.234 & 0.238 & \multirow{2}{*}{0.237} & \multirow{2}{*}{0.234} \\
\hline & Randomdec & 0.236 & 0.237 & 0.235 & 0.238 & & \\
\hline \multirow{2}{*}{2} & Original & 0.729 & 0.727 & 0.727 & 0.729 & \multirow{2}{*}{0.727} & \multirow{2}{*}{0.730} \\
\hline & Randomdec & 0.727 & 0.727 & 0.727 & 0.726 & & \\
\hline \multirow{2}{*}{3} & Original & 1.272 & 1.268 & 1.268 & 1.277 & \multirow{2}{*}{1.271} & \multirow{2}{*}{1.266} \\
\hline & Randomdec & 1.271 & 1.270 & 1.266 & 1.277 & & \\
\hline \multirow{2}{*}{4} & Original & 1.588 & 1.588 & 1.582 & 1.596 & \multirow{2}{*}{1.588} & \multirow{2}{*}{1.596} \\
\hline & Randomdec & 1.586 & 1.589 & 1.584 & 1.592 & & \\
\hline \multirow{2}{*}{5} & Original & 2.709 & 2.712 & 2.723 & 2.719 & \multirow{2}{*}{2.721} & \multirow{2}{*}{-} \\
\hline & Randomdec & 2.727 & 2.722 & 2.719 & 2.738 & & \\
\hline \multirow{2}{*}{6} & Original & 3.471 & 3.481 & 3.453 & 3.467 & \multirow{2}{*}{3.468} & \multirow{2}{*}{ - } \\
\hline & Randomdec & -- & -- & - & - & & \\
\hline \multirow{2}{*}{7} & Original & 5.432 & 5.422 & 5.434 & 5.424 & \multirow{2}{*}{5.428} & \multirow{2}{*}{ - } \\
\hline & Randomdec & - & - & - & - & & \\
\hline
\end{tabular}

Original: It is read from FFT plot of averaged acceleration (number of data points = 8192)

Randomdec: It is estimated by applying the circle fitting method on randomdec signals.

ratios, which are very sensitive to the smoothness of the Fourier transformed response functions near the resonant frequencies, can be estimated more accurately utilizing the randomdec signals. In this study, the damping ratios for the first four modes of the structural system were estimated from the randomdec signals by the half-power bandwidth method. The estimated values are summarized in Table 2, along with other experimental results from Tang et al. (1995). The estimated

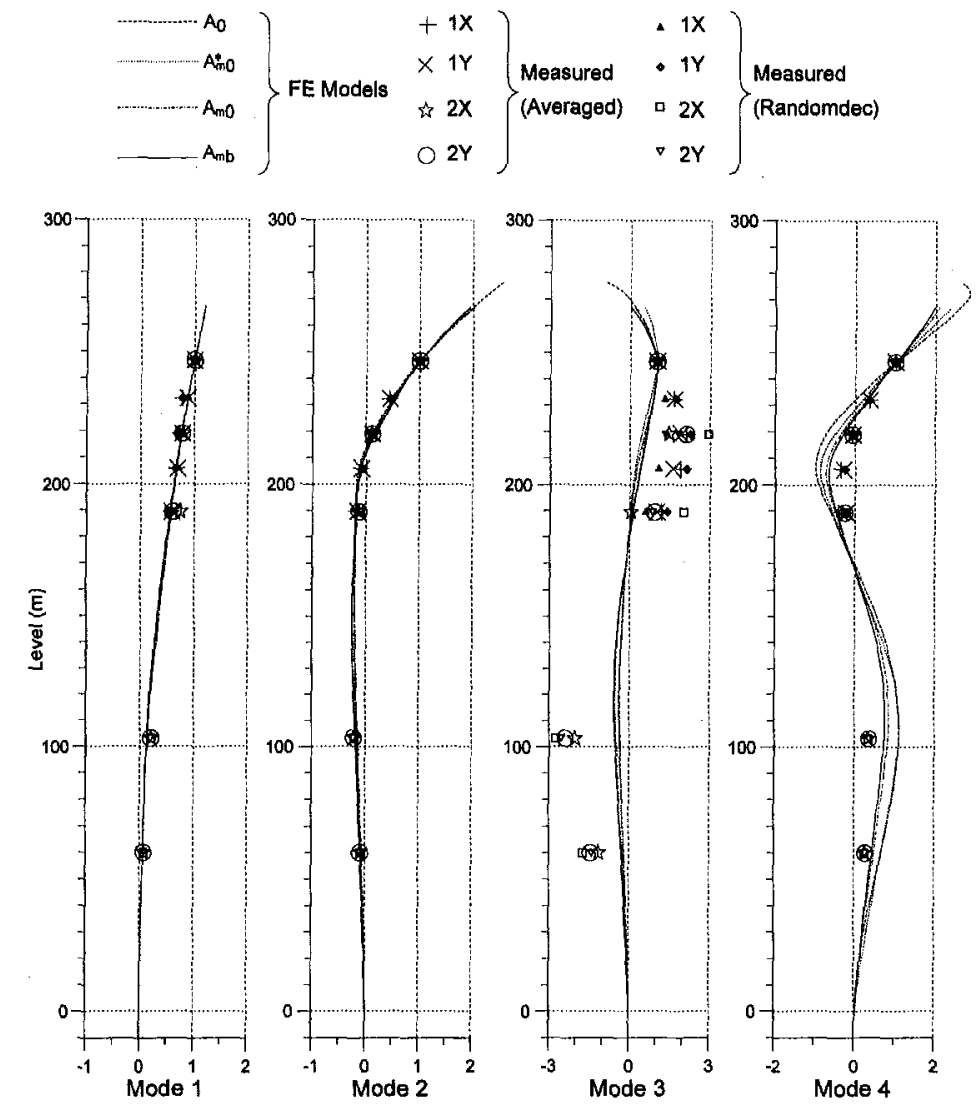

Fig. 6 Calculated and measured mode shapes 
Table 2 Estimated damping ratios of the Nanjing TV Tower utilizing randomdec signals

\begin{tabular}{|c|c|c|c|c|c|}
\hline \multirow{2}{*}{\multicolumn{2}{|c|}{ Used Record }} & \multicolumn{4}{|c|}{ Estimated Damping Ratio (\%) } \\
\hline & & mode 1 & mode 2 & mode 3 & mode 4 \\
\hline \multirow{5}{*}{$1 \mathrm{X}$} & 5 & 1.298 & 1.585 & - & -- \\
\hline & 4 & 1.302 & 1.591 & 0.544 & - \\
\hline & 3 & 1.326 & 0.746 & 0.584 & 0.680 \\
\hline & 2 & 1.330 & 1,420 & 0.596 & - \\
\hline & 1 & 1.355 & 1.661 & 0.596 & - \\
\hline \multirow{5}{*}{$I Y$} & 5 & 1.700 & 0.396 & -- & - \\
\hline & 4 & 1.715 & 0.396 & - & -- \\
\hline & 3 & 1.725 & 0.397 & - & -- \\
\hline & 2 & 1.731 & 0.394 & - & -- \\
\hline & 1 & 1.787 & 0.390 & - & -- \\
\hline \multirow{5}{*}{$2 X$} & 5 & 1.273 & 0.385 & 0.748 & 0.431 \\
\hline & 3 & 1.285 & 0.389 & - & 0.433 \\
\hline & 1 & 1.308 & 0.389 & - & 0.410 \\
\hline & 2 & 1.268 & 0.387 & $\cdots$ & 0.434 \\
\hline & 4 & 1.396 & 0.437 & - & 0.392 \\
\hline \multirow{5}{*}{$2 Y$} & 5 & 2.036 & 0.504 & 0.761 & 0.607 \\
\hline & 3 & 2.518 & 0.502 & 0.623 & 0.534 \\
\hline & 1 & 2.178 & 0.512 & 0.624 & 0.558 \\
\hline & 2 & 2.490 & 0.500 & 0.601 & 0.515 \\
\hline & 4 & 2.231 & 0.473 & -- & 0.534 \\
\hline \multicolumn{2}{|c|}{$\begin{array}{l}\text { This Study } \\
\text { (Average) }\end{array}$} & 1.663 & 0.677 & 0.631 & 0.503 \\
\hline \multicolumn{2}{|c|}{ Tang } & 1.480 & 1.348 & 0.887 & 0.760 \\
\hline
\end{tabular}

damping rations were 1.66 percent for the first mode and less than one percent for the other modes, which are comparable with typical values observed in similar structures (Bao and Ko, 1991).

3.4 Mode Shapes. If the estimates of natural frequencies are accurate, corresponding mode shapes at the measured points can be easily obtained using power spectral density functions, as follows (Bao and Ko, 1991; Gavin et al., 1992):

$$
\begin{gathered}
\hat{\Phi}_{p k}^{(l)}=\frac{S_{y_{p} y_{q}}\left(f_{k}\right)}{S_{y_{q} y_{q}}\left(f_{k}\right)} \\
\hat{\Phi}_{p k}^{(I)}=\frac{S_{y_{p} y_{p}}\left(f_{k}\right)}{S_{y_{p} y_{q}}\left(f_{k}\right)} \\
\hat{\Phi}_{p k}^{(I I)}=\operatorname{sign}\left(\hat{\Phi}_{p k}^{(l)}\right) \sqrt{\frac{S_{y_{p} y_{p}}\left(f_{k}\right)}{S_{y_{q} y_{q}}\left(f_{k}\right)}}
\end{gathered}
$$

in which $\hat{\Phi}_{p k}^{(I)}, \hat{\Phi}_{p k}^{(I I)}$, and $\hat{\Phi}_{p k}^{(I I I)}$ are estimates of the $k$ th mode shape at point $p$ normalized to the amplitude at reference point $q ; S_{y_{p} y_{p}}$ and $S_{y_{p} y_{q}}$ are respectively auto and cross-spectral density functions; $f_{k}$ is the $k$ th natural frequency; and sign $(\cdot)$ is a sign function which equals to 1 or -1 . Assuming the measured response $y_{p}(t)$ at point $p$ can be separated into uncorrelated pure response $\hat{y}_{p}(t)$ and noise $n_{p}(t)$, Eq. (4) can be rewritten as

$$
\begin{gathered}
\hat{\Phi}_{p k}^{(I)}=\hat{\Phi}_{p k} \frac{1}{1+S_{n_{q} n_{q}}\left(f_{k}\right) / S_{\hat{y}_{q} \hat{\vartheta}_{q}}\left(f_{k}\right)} \\
\hat{\Phi}_{p k}^{(I I)}=\hat{\Phi}_{p k}\left(1+\frac{S_{p_{p} n_{p}}\left(f_{k}\right)}{S_{\hat{y}_{p} \hat{y}_{p}}\left(f_{k}\right)}\right) \\
\hat{\Phi}_{p k}^{(I I I)}=\hat{\Phi}_{p k} \sqrt{\frac{1+S_{n_{p} n_{p}}\left(f_{k}\right) / S_{\hat{y}_{p_{p}} \hat{y}_{p}}\left(f_{k}\right)}{1+S_{n_{q} n_{q}}\left(f_{k}\right) / S_{\hat{y}_{q} \hat{y}_{q}}\left(f_{k}\right)}}
\end{gathered}
$$

where $\hat{\Phi}_{p k}=S_{\hat{y}_{p} \hat{y}_{q}}\left(f_{k}\right) / S_{\hat{y}_{q} \hat{y}_{q}}\left(f_{k}\right)$. Equation (5c) shows that the mode shape can be identified accurately regardless of noise level, if the noise levels at all the measured locations are proportional to the corresponding intensity levels of records. Because $\left|\hat{\Phi}_{p k}^{(I)}\right| \leq\left|\hat{\Phi}_{p k}\right| \leq\left|\hat{\Phi}_{p k}^{(I I)}\right|$ and $\left|\hat{\Phi}_{p k}^{(I)}\right| \leq\left|\hat{\Phi}_{p k}^{(I I)}\right| \leq\left|\hat{\Phi}_{p k}^{(I I)}\right|$, the effects of noise on the mode shape estimation can also be examined by evaluating the difference between the lower bound $\hat{\Phi}_{p k}^{(I)}$ and the upper bound $\hat{\Phi}_{p k}^{(I)}$ of the mode shape estimates.

The first four mode shapes were identified from the power spectral density functions of the randomdec signals and acceleration records averaged using the conventional approach with 4096 points segments and 30 percent overlap. The Ch. 5 location ( small observation hall) was chosen as the reference point $q$. The estimated results by Eq. $(5 c)$ are shown in Fig. 6 (compared with those calculated by the identified mass and stiffness matrices to be described in the next section). Despite of the fact that the input loads on the structural system were not possible to measure, the estimated mode shapes appear reasonable. In addition, the differences between the lower bound and the upper 
Table 3 Nodal coordinates, lumped masses, and stiffness parameters of baseline finite element model $A_{0}$

\begin{tabular}{|c|c|c|c|c|}
\hline $\begin{array}{l}\text { Node } \\
\text { Number }\end{array}$ & $\begin{array}{l}\text { Lumped Mass } \\
\qquad(\mathrm{Mg})\end{array}$ & $\begin{array}{l}\text { Element } \\
\text { Number }\end{array}$ & $\begin{array}{l}\text { Cross Sectional } \\
\text { Area }\left(\mathrm{m}^{2}\right)\end{array}$ & $\begin{array}{l}\text { Moment of } \\
\text { Inertia }\left(\mathrm{m}^{4}\right)\end{array}$ \\
\hline 0 & - & \multirow{2}{*}{1} & \multirow{2}{*}{53.22} & \multirow{2}{*}{3695.5} \\
\hline 1 & 3992.90 & & & \\
\hline 2 & 3186.74 & 2 & 47.95 & 2707.4 \\
\hline 3 & 2820.10 & 3 & 42.69 & 1938.7 \\
\hline 4 & 2319.79 & 4 & 38.89 & 1457.4 \\
\hline 5 & 1917.95 & 5 & 32.77 & 1114.2 \\
\hline 6 & 1624.51 & 6 & 30.79 & 883.0 \\
\hline 7 & 1628.07 & 7 & 29.57 & 792.6 \\
\hline 8 & 1322.34 & 8 & 27.90 & 673.0 \\
\hline 9 & 3395.32 & 9 & 25.31 & 568.5 \\
\hline 10 & 5678.60 & 10 & 47.26 & 1134.7 \\
\hline 11 & 1325.79 & 11 & 47.26 & 465.9 \\
\hline 12 & 359.10 & 12 & 7.386 & 27.78 \\
\hline 13 & 1081.47 & 13 & 7.386 & 27.78 \\
\hline 14 & 165.13 & 14 & 4.96 & 8.08 \\
\hline 15 & 18.70 & 15 & 0.2124 & $1.10930 \mathrm{e}-01$ \\
\hline 16 & 12.00 & 16 & 0.1075 & $2.07160 \mathrm{e}-02$ \\
\hline 17 & 4.00 & 17 & 0.0584 & $5.19079 \mathrm{e}-03$ \\
\hline
\end{tabular}

bound of the mode shape estimates were less than one percent in the present ambient vibration records. Therefore, the noise effects can be neglected in this analysis.

\section{Finite Element Model Correlated With Measure- ments}

4.1 Baseline Finite Element Model. A two-dimensional baseline finite element model for the tower structural system, namely $A_{0}$, is constructed based on design drawings of the structure and the finite element model developed by Cheng (1992). The model consists of 17 beam elements and lumped masses. The elevation of the finite element model is shown in Fig. 2. The element properties of the baseline model are shown in Table 3. In this model, tapered beams were replaced by equivalent piece-wise uniform prismatic beams for the analysis purpose. The cross-sectional area and moment of inertia were taken at the midsection of each element. Lumped masses were assumed to be the same as Cheng's model. The Young's moduli for the finite elements used in analysis are: $E_{c}=3.237 \times 10^{7}$ $\mathrm{KPa}$ for concrete members, and $E_{s}=2.060 \times 10^{8} \mathrm{KPa}$ for steel members.

\subsection{Method of Updating Baseline Model.}

Definition of Updated Model. Updated mass and stiffness matrices can be written in the form

$$
\begin{aligned}
\hat{\mathbf{M}} & =\mathbf{M}+\Delta \mathbf{M} \\
\hat{\mathbf{K}} & =\mathbf{K}+\Delta \mathbf{K}
\end{aligned}
$$

where $\mathbf{M}$ and $\mathbf{K}$ are the baseline mass and stiffness matrices; $\Delta \mathbf{M}$ and $\Delta \mathbf{K}$ are correction mass and stiffness matrices. It is assumed that the updated mass matrix is the same as the baseline mass matrix, and the correction stiffness matrix is represented by a linear combination of the element stiffness matrices in the baseline model

$$
\begin{gathered}
\Delta \mathbf{M}=\mathbf{0} \\
\Delta \mathbf{K}=\sum_{e=1}^{N_{e}} a_{e} \mathbf{K}_{e}
\end{gathered}
$$

in which $a_{e}$ is correction coefficient of flexural stiffness $E I$ for the $e$ th element; $N_{e}$ denotes the number of finite elements; and $\mathbf{K}_{e}$ is the $e$ th element stiffness matrix of the baseline.

Inverse Modal Perturbation Theory. The sensitivities of the natural frequencies and mode shapes to the stiffness correction coefficients were obtained from the second-order inverse modal perturbation theory (Yun and Hong, 1992), as follows:

$$
\begin{gathered}
\hat{\lambda}_{k}=\lambda_{k}+\frac{\mathbf{s}_{k k} \mathbf{a}}{\mu_{k}\left(1+D_{k k}\right)} \\
\left\{\hat{\Psi}_{k}\right\}=\mathbf{T}_{k} \mathbf{S}_{k} \mathbf{a}
\end{gathered}
$$

where

$$
\begin{gathered}
S_{k}(j, e)=\left\{\Psi_{j}\right\}^{T} \mathbf{K}_{e}\left\{\hat{\Psi}_{k}\right\} \\
T_{k}(i, j)=\frac{\Psi_{i j}}{\mu_{j}\left(\hat{\boldsymbol{\lambda}}_{k}-\lambda_{j}\right)} \\
1+D_{k k}=\sqrt{\hat{\mu}_{k}-\sum_{j=1, j \neq k}^{N_{\text {mode }}} \mu_{j} D_{k j}^{2}} \\
D_{k j}=\frac{\mathbf{s}_{k j} \mathbf{a}}{\mu_{j}\left(\hat{\boldsymbol{\lambda}}_{k}-\lambda_{j}\right)} \\
\mu_{k}=\left\{\Psi_{k}\right\}^{T} \mathbf{M}\left\{\Psi_{k}\right\}, \quad \hat{\mu}_{k}=\left\{\hat{\Psi}_{k}\right\}^{T} \mathbf{M}\left\{\hat{\Psi}_{k}\right\} .
\end{gathered}
$$

$\lambda_{k}$ and $\hat{\lambda}_{k}$ are, respectively, the square of the $k$ th natural frequencies from baseline and updated models; $N_{\text {mode }}$ is the number of modes used; $\left\{\Psi_{k}\right\}$ and $\left\{\hat{\Psi}_{k}\right\}$ are, respectively, the $k$ th mode shape vectors of baseline and updated models; $\mathbf{s}_{k j}$ is the $j$ th row vector of matrix $\mathbf{S}_{k}$; and $\mathbf{a}$ is the stiffness correction coefficient vector. 
Quadratic Optimization. From Eq. (10), an expected difference in the normalized strain energy of the $k$ th mode between an updated model and the measurement can be written as

$$
e_{k}(\mathbf{a})=\frac{1}{\tilde{\lambda}_{k}}\left\{\mathbf{s}_{k k} \mathbf{a}-\mu_{k}\left(1+D_{k k}\right) \Delta \lambda_{k}\right\}
$$

where $\Delta \lambda_{k}$ is a difference in the square of $k$ th natural frequency between the baseline and measurement $\left(=\tilde{\lambda}_{k}-\lambda_{k}\right)$; and $\tilde{\lambda}_{k}$ denotes the square of measured natural frequency of the $k$ th mode. Now, the following objective function is established and minimized to obtain the optimal value of a,

$$
\min _{\mathbf{a}} J=\sum_{k=1}^{N_{k}}\left\{w_{k} e_{k}(\mathbf{a})\right\}^{2},
$$

in which $N_{k}$ is the number of measured natural frequencies, and $w_{k}$ is a weighting factor for the $k$ th mode that may be assumed as the amplitude of Fourier transformed response function at the $k$ th natural frequency.

Measured mode shapes are considered in constraining equations with proper error bounds, rather than being involved in the objective function, since their accuracy is worse than that of natural frequencies. Moreover, since it is impossible to measure the ambient vibration at all nodal points in practice for a large-scale structural system, it is very difficult to define an objective function in conjunction with the natural frequencies systematically. Thus the relative values of mode shapes were incorporated into constraining equations in this study. Eventually, the constraining equations related to the $k$ th mode shape is expressed as

$\left|\frac{\hat{\Psi}_{p k}(\mathbf{a})}{\hat{\Phi}_{q k}}-\frac{\hat{\Psi}_{q k}(\mathbf{a})}{\hat{\Phi}_{q k}}\right| \leq \epsilon_{k}$

$$
\left(p=1,2, \ldots, N_{o b} ; \text { but } p \neq q\right)
$$

where $\hat{\Phi}_{p k}$ is the $k$ th measured mode shape at point $p$; and $\epsilon_{k}$ is an error bound for the $k$ th mode shape. In addition to the constraints, upper and lower bounds for the stiffness correction coefficient vector $\mathbf{a}$, which can be determined by engineering judgment, are added to the constraints to reflect real conditions of the structure. Because the measurement points did not coincide with the nodal points of the finite element model in this study, the constraining equations were applied after representing the mode shape data by the corresponding nodal responses and shape functions of the beam element.

In summary, the system identification problem to establish an appropriate dynamic model correlating with measured modal parameters can be represented as an optimization problem to determine the stiffness correction coefficients under constraining equations, as follows:

Find out vector a which minimizes the following objective function:

$$
J=\frac{1}{2} \mathbf{a}^{T} \mathbf{H a}+\mathbf{f}^{T} \mathbf{a}
$$

subject to conditions as

$$
\mathbf{G a} \leq \mathbf{b} \text { and } \quad \mathbf{a}_{\min } \leq \mathbf{a} \leq \mathbf{a}_{\max }
$$

where $\mathbf{H}$ and $\mathbf{f}$ are derived from Eqs. (17) and (18), and $\mathbf{G}$ and $\mathbf{b}$ are from Eqs. (11) and (19).

Because the matrices $\mathbf{H}, \mathbf{f}$, and $\mathbf{G}$ are dependent on parameter a, the solution should be found by iteration.

\subsection{Updated Finite Element Model of Nanjing TV Tower.}

Better Baseline Finite Element Model. It is well known that the convergence and accuracy of an optimization solution depend on the initial baseline estimates. In this study, an analytical model, namely $A_{b}$, was developed as a better baseline (com-

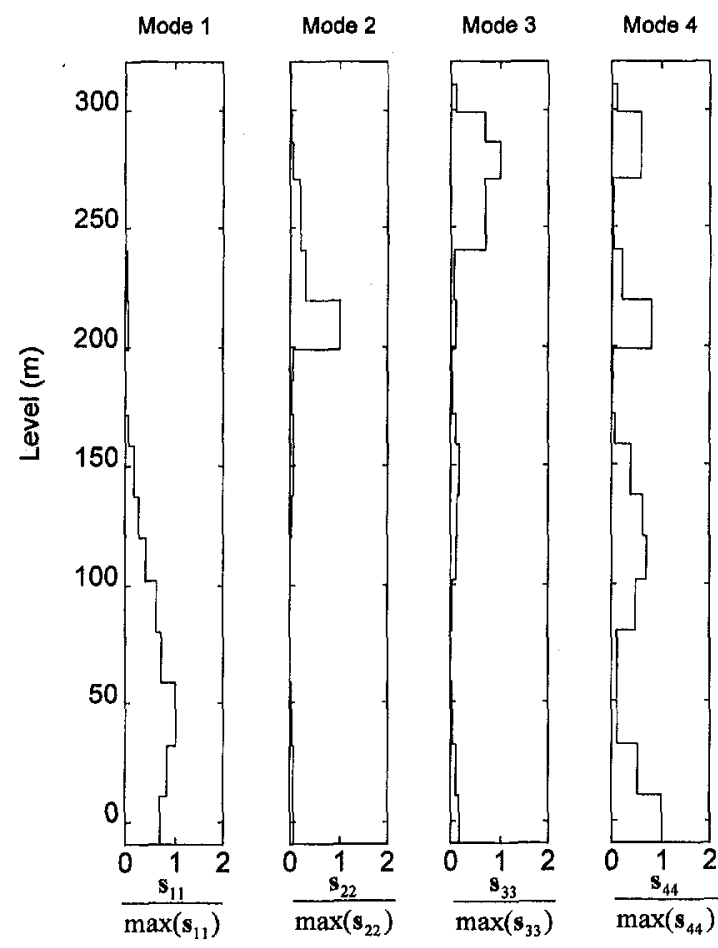

Fig. 7 Normalized element modal strain energy diagram

pared with model $A_{0}$ mentioned in Section 4.1). The normalized element modal strain energy diagram $\left(\mathbf{s}_{k k} / \max \left(\mathbf{s}_{k k}\right)\right)$, which represents the contribution of an element stiffness to the $k$ th natural frequency, was produced in order to get insight on how to modify the element stiffness to reduce the difference in natural frequencies between the calculated and the measured. The diagram for the first four modes of the initial baseline model $A_{0}$ is shown in Fig. 7. From the figure, it can be seen that stiffness correction coefficients for the lower part of the structure, say elements below level of $100 \mathrm{~m}$, shall be positive to increase natural frequency for the first mode of updated model, while those for the upper part of the structure for the second and third modes. Therefore, the stiffness correction coefficient vector can be determined by trial and error referring to the diagram and comparing to the recalculated and measured natural frequencies. Natural frequencies by this better baseline are listed in Table 6, which show a much better agreement with the measured ones than those by the baseline.

Updated Model. Three updated models $A_{m 0}^{*}, A_{m 0}$, and $A_{m b}$ were obtained starting from baseline models $A_{0}$ and $A_{b}$. The model $A_{m 0}^{*}$ has been updated by using the measured natural frequencies only, while models $A_{m 0}$, and $A_{m b}$ were identified utilizing the measured mode shape data as well as the natural frequencies. The brief descriptions regarding the models are given in Table 4. Seven natural frequencies and four mode shapes identified on the basis of the measurement data were used in updating the finite element models.

In the optimization process, the degree of convergence and accuracy of solutions are fairly sensitive to the upper and lower bounds of the stiffness correction factor ( $\mathbf{a}_{\min }$ and $\mathbf{a}_{\max }$ ), the error bounds for the mode shape data $\left(\epsilon_{k}\right)$ and weighting factors $\left(w_{k}\right)$ in the objective function. The various parameters used in the optimization process are given in Table 5. Convergence of the natural frequencies is demonstrated in Fig. 8. The converged stiffness correction coefficients are summarized in Table 5 and Fig. 9. As shown in Fig. 9, the stiffness scale factors of different updated models are very similar to each other, independent of the baseline model and information used. In particular, models 
Table 4 Description on baseline and updated finite element models

\begin{tabular}{|c|c|c|c|}
\hline Model Name & Base Model & $\begin{array}{l}\text { Construction } \\
\text { Method }\end{array}$ & Information Used \\
\hline Ao & $\begin{array}{c}\text { Cheng's 3D } \\
\text { model }\end{array}$ & & Design drawings, 3D model \\
\hline$A *$ mo & $\overrightarrow{A_{0}}$ & $\mathrm{IMP}^{*}$ & 7 measured natural frequencies \\
\hline$A_{\text {mo }}$ & $A_{0}$ & IMP* & $\begin{array}{c}7 \text { measured natural frequencies } \\
\text { and } 4 \text { mode shapes }\end{array}$ \\
\hline$A_{b}$ & Ao & trial and error & $\begin{array}{c}\text { Element modal strain energy diagram } \\
\text { (Fig. 7) }\end{array}$ \\
\hline$A_{\mathrm{mb}}$ & $A_{b}$ & IMP* & $\begin{array}{c}7 \text { measured natural frequencies } \\
\text { and } 4 \text { mode shapes }\end{array}$ \\
\hline
\end{tabular}

Note: * Inverse Modal Perturbation

$A_{m 0}$ and $A_{m b}$ are nearly identical despite of their quite different baseline models. The updated stiffness models appear stiffer than the baseline model, especially in the location of small and large observation hall structures and lower part of the main three-leg structure. These may result from difference of concrete stiffness between the baseline and the real structure, and additional stiffening effects by prestressing forces, reinforced steel, and nonstructural components.

The natural frequencies and mode shapes calculated from the updated models are shown in Table 6 and Fig. 6, respectively. The first five natural frequencies show excellent agreement with the measured results. The corresponding mode shapes also match very well with the measured ones except the third mode which is, however, not a critical mode for the response at the small observation hall.

Although a better baseline model, such as $A_{b}$ in this study, may improve the convergence, a conventional model made from the design drawings of the structure, such as $A_{0}$ in this study, appears to be a reasonable baseline for establishing the updated model, if sufficient information for the real structure can be incorporated in the optimization process. In general, the updated models $A_{m 0}$ and $A_{m b}$ constructed in conjunction with both the

Table 5 Stiffness correction coefficients of better baseline and updated finite element models

\begin{tabular}{|c|c|c|c|c|}
\hline \multirow{2}{*}{$\begin{array}{c}\text { Element } \\
\text { Number }\end{array}$} & \multicolumn{4}{|c|}{ Stiffness Correction Coefficient $\left(a_{e}\right)^{*}$} \\
\cline { 2 - 5 } & $\mathrm{A}_{\mathrm{b}}$ & $\mathrm{A}^{*}{ }_{\mathrm{m} 0}$ & $\mathrm{~A}_{\mathrm{m} 0}$ & $\mathrm{~A}_{\mathrm{mb}}$ \\
\hline 1 & 0.1 & -0.300 & -0.262 & -0.300 \\
\hline 2 & 2.0 & 3.000 & 3.000 & 2.349 \\
\hline 3 & 1.5 & 3.000 & 3.000 & 3.000 \\
\hline 4 & 1.5 & 3.000 & 3.000 & 3.000 \\
\hline 5 & -0.2 & 0.047 & 0.757 & 0.779 \\
\hline 6 & -0.3 & -0.400 & -0.400 & -0.400 \\
\hline 7 & -0.3 & -0.400 & -0.400 & -0.400 \\
\hline 8 & -0.2 & 3.000 & -0.400 & -0.400 \\
\hline 9 & 0.1 & 3.000 & 3.000 & 3.000 \\
\hline 10 & 1.5 & 4.000 & 4.000 & 4.000 \\
\hline 11 & 1.1 & 3.000 & -0.400 & -0.400 \\
\hline 12 & 0.2 & 0.016 & 0.116 & 0.174 \\
\hline 13 & 0.5 & 4.000 & 4.000 & 4.000 \\
\hline 14 & 0.4 & 0.162 & 0.191 & 0.154 \\
\hline 15 & 0.0 & 0.085 & 0.150 & 0.150 \\
\hline 16 & 0.0 & -0.150 & -0.088 & -0.031 \\
\hline 17 & 0.0 & -0.150 & -0.150 & -0.150 \\
\hline
\end{tabular}

Note: * The updated element stiffness matrix shall be $\left(1+\mathrm{a}_{\mathrm{e}}\right) \mathrm{K}_{\mathrm{e}}$, in which $\mathrm{K}_{\mathrm{e}}$ is the $e$-th element stiffness matrix of baseline model. The coefficients used for optimization are as follows: $w_{1}=w_{2}=100, w_{3}=w_{4}=4, w_{5}=1$, $w_{6}=w_{7}=0.001 ;-0.3 \leq a_{1} \leq 3.0,-0.3 \leq a_{10} \leq 4.0,-0.4 \leq a_{13} \leq 4.0,-0.3 \leq a_{e} \leq 3.0$ for other concrete member, and $-0.15 \leq a_{e} \leq 0.15$ for steel members; $\varepsilon_{1}=0.0075$ and $\varepsilon_{k}=0.01$ for the other modes. 

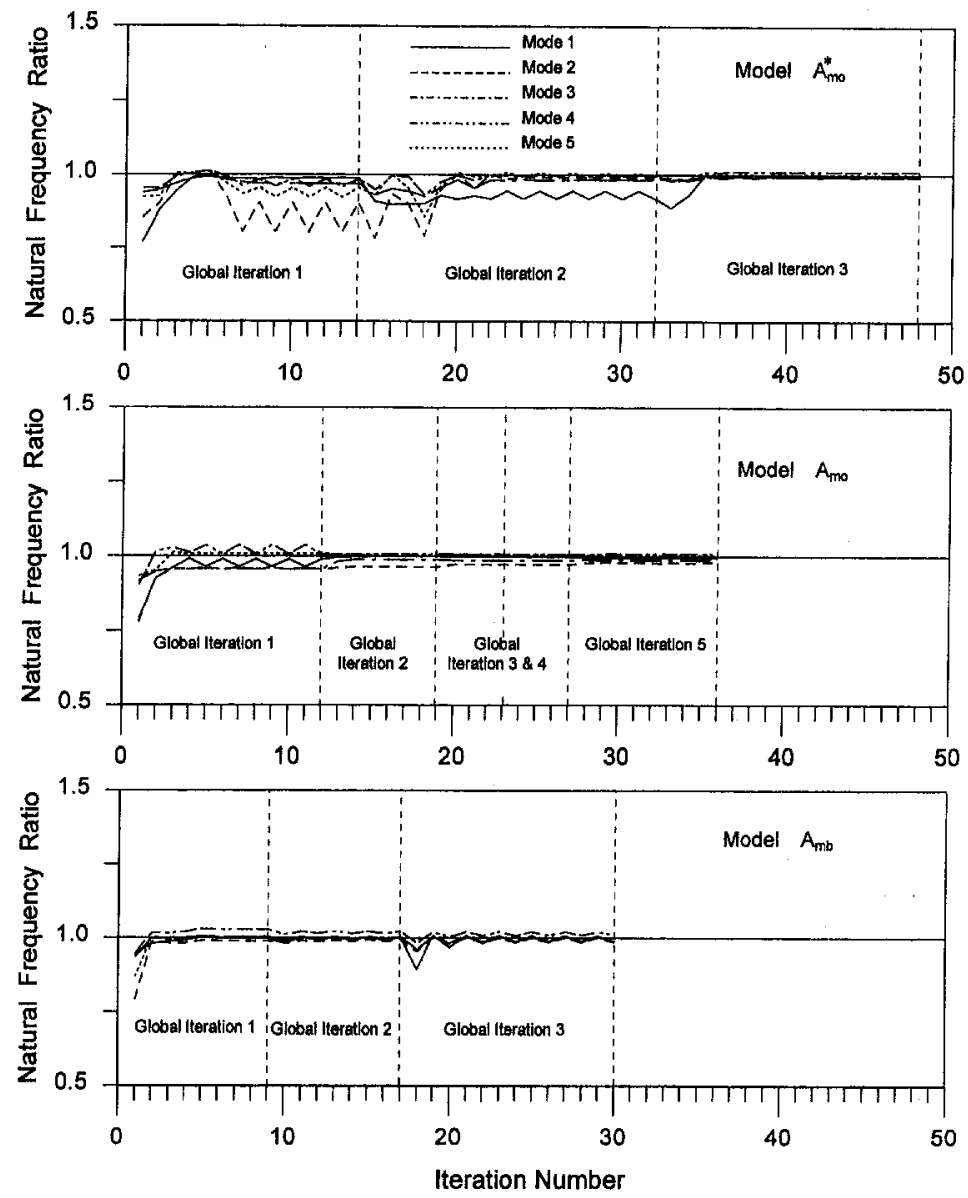

Fig. 8 Convergence of natural frequencies of updated finite element models
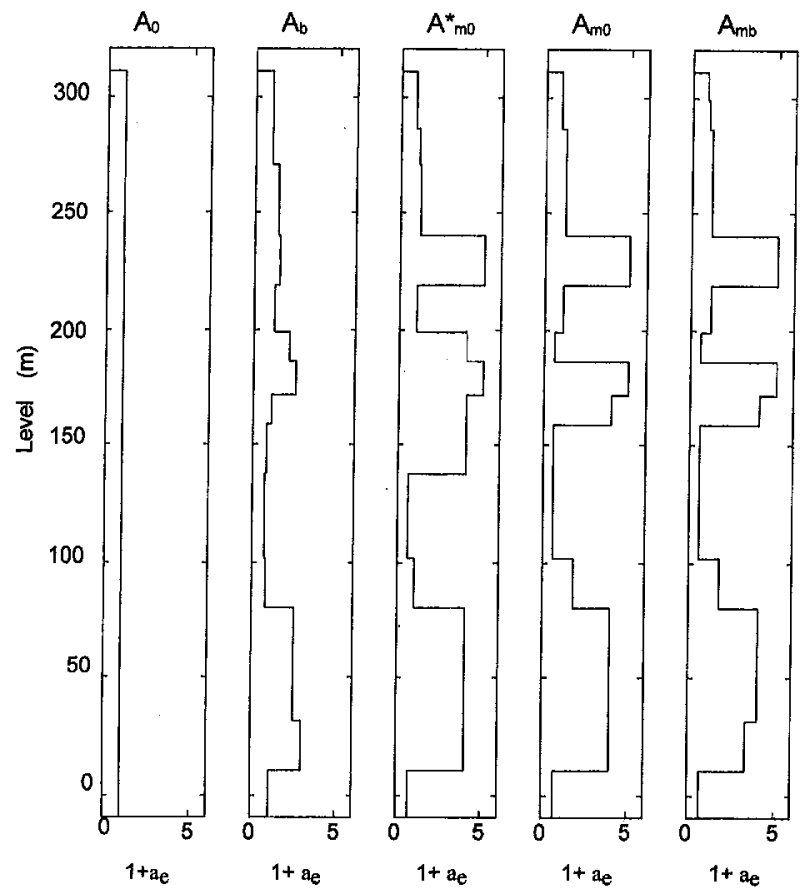

Fig. 9 Element stiffness scale factor $\left(1+a_{0}\right)$ of baseline and updated finite element models

1020 / Vol. 65, DECEMBER 1998

measured natural frequencies and mode shapes show better agreement with the measured mode shapes than the updated model $A_{m 0}^{*}$ using natural frequency information only.

\section{Conclusion}

Ambient vibration tests were performed on a large-scale structural system using the newly developed optical fiber accelerometers, in order to establish an analytical model of the structure for designing an active mass damper system to control the structural vibration under wind excitations. Modal parameters including natural frequencies, damping ratios, and mode shapes were estimated from the measured acceleration records by using various system identification techniques, among which the random decrement technique was found to be particularly effective in identifying the damping ratios due to the removal of random wind effects from the measured signals. More importantly, several finite element stiffness matrices involving a large number of elements were successfully estimated taking advantage of the very limited ambient vibration information obtained from a limited number of sensors. The second-order inverse modal perturbation theory was effective in establishing the sensitivity relationship between the element stiffness and the modal parameters, which was convenient used in the quadratic optimization to derive the system stiffness matrix producing the minimum difference between the calculated and measured modal strain energy. In the process of this optimization, it was found reasonable to consider the mode shape information as a constraint, rather than embedding it together with the natural frequency information in the objective function. The finite element models thus identified represent the actual structural system very well, as their calculated dynamic characteristics satisfactorily 


\begin{tabular}{|c|c|c|c|c|c|c|}
\hline \multirow{2}{*}{$\begin{array}{c}\text { Mode } \\
\text { Number }\end{array}$} & \multicolumn{5}{|c|}{ Calculated using Finite Element Model } & \multirow{2}{*}{ Measured } \\
\cline { 2 - 6 } & $\mathrm{A}_{\circ}$ & $\mathrm{A}_{\mathrm{b}}$ & $\mathrm{A}_{\mathrm{mo}}$ & $\mathrm{A}_{\mathrm{mo}}$ & $\mathrm{A}_{\mathrm{mb}}$ & \\
\hline 1 & 0.199 & 0.228 & 0.235 & 0.236 & 0.233 & 0.237 \\
\hline 2 & 0.628 & 0.716 & 0.727 & 0.711 & 0.716 & 0.727 \\
\hline 3 & 1.228 & 1.285 & 1.264 & 1.257 & 1.256 & 1.271 \\
\hline 4 & 1.625 & 1.681 & 1.605 & 1.600 & 1.602 & 1.588 \\
\hline 5 & 2.489 & 2.798 & 2.726 & 2.730 & 2.721 & 2.721 \\
\hline 6 & 4.153 & 4.385 & 4.622 & 4.605 & 4.611 & 3.468 \\
\hline 7 & 4.705 & 4.822 & 5.461 & 4.868 & 4.864 & 5.428 \\
\hline
\end{tabular}

matched the observed ones from the ambient vibration test actually performed on the large-scale structural system.

\section{Acknowledgments}

The authors wish to thank Prof. C.-B. Yun and Dr. H.-J. Lee at the Korea Advanced Institute of Science and Technology for their valuable comments on the system identification results. This work was supported by the National Science Foundation under the CAREER Award and monitored by Dr. N. Sabadell, Program Director. The second author thanks, for the partial financial support, the Korea Science and Engineering Foundation on his postdoctoral research related to this work.

\section{References}

Ansari, F, ed., 1993, Applications of Fiber Optic Sensors in Engineering Me chanics, ASCE, Reston, VA.

Bao, Z. W., and Ko, J. M., 1991, "Determination of modal parameters of tall buildings with ambient vibration measurements," The International Journal of Analytical and Experimental Modal Analysis, Vol. 6, No. 1, pp. 57-68.

Cheng, W. R., 1992, "The analysis of dynamic characteristics of Nanjing TV

Tower," Technical Report of Southeast University, in Chinese.

Cole, H. J., 1973, "On-line failure detection and damping measurement of aerospace structures by random decrement signatures," NASA Cr-2205.

Ding, Dajun, and Ren, Zuenhua, 1994, "Design testing and analysis of the

Nanjing TV Tower,' Concrete International, Nov., pp. 42-44.

Ewins, D. J., 1984, Modal Testing: Theory and Practice, John Wiley and Sons, New York.

Feng, M. Q., 1996, "An experimental study on electro-optical displacement sensors," Nondestructive Test and Evaluation, Vol. 13, pp. 5-14.

Gavin, H., Suan, S., Grossman, J., Pekelis, E, and Jacob, K., 1992, "Lowlevel dynamic characteristics of four tall flat-plate buildings in New York City,' NCEER Technical Report, NCEER-92-0034.

Hong, K.-S., and Yun, C.-B., 1992, "Improved Method for Frequency Domain Identification of Structures," Engineering Structures, Vol. 15, No. 3, pp. 179188.

Hoshiya, M., and Sutoh, A., 1993, "Kalman filter-finite element method in identification," Journal of Engineering Mechanics, ASCE, Vol. 119, No. 2, pp. $197-210$.
Ibrahim, S. R., 1977, "Random decrement technique for modal identification of structures," Journal of Spacecraft and Rocket, AIAA, Vol. 14, No. 11, pp. $696-700$.

Kersey, A. D., 1994, "Role of fiber optic sensors in intelligent infrastructure systems," Proc. the First World Conference on Structural Control, Los Angeles, Aug., Plenary-3-9.

Okuma, M., 1993, "Correction of finite element models using experimental modal data for vibration analysis," Finite Elements in Analysis and Design, Vol. 14 , pp. $153-162$.

Shinozuka, M., and Ghanem, R., 1995, "Structural system identification I: Theory," Journal of Engineering Mechanics, ASCE, Vol. 121, No. 2, pp. 255264

Stetson, K. A., and Harrison, I. R,, 1981, "Redesign of structural vibration modes by finite-element inverse perturbation," ASME Joumal of Engineering for Power, Vol. 103, pp. 319-324

Stetson, K. A., and Palma, G. E., 1975, "Inversion of first-order perturbation theory and its application to structural design," AIAA Journal, Vol. 14, No. 4, pp. $454-460$.

Tang, J. X., Li, L., and Wu, C. Y., 1995, "Report on field measurement of Nanjing TV Tower," Technical Report of Southeast University, in Chinese.

Ting, T., and Ojalvo, I. U., 1989, "Dynamic structural correction via nonlinear programming technique," Finite Elements in Analysis and Design, Vol. 5, pp. $247-256$.

Torkamani, M. A. M., and Ahmadi, A. K., 1988, "Stiffness identification of a tall building during construction period using ambient tests,' Earthquake Engineering and Structural Dynamics, Vol. 16, pp. 1177-1188.

Tsai, W. H., and Yang, J. C. S., 1988, "Non-destructive Evaluation of Composite Structures Using System Identification Technique," ASME Journal of Engineering Materials and Technology, Vol. 110, pp. 134-139.

Vries, M. D., Murphy, K. A., Poland, S., Arya, V., Claus, R. O., and Masri, S. F., 1994, "Optical fiber sensor for civil structure applications: a comparative evaluation," Proc. the First World Conference on Structural Control, Los Angeles, CA, Aug., WA3-23-29.

Wang, D., and Haldar, A., 1994, "Element-level system identification with unknown input," Journal of Engineering Mechanics, ASCE, Vol. 120, No. 1, pp. 159-176.

Yang, J. C. S., Chen, J., and Dagalakis, N. G., 1984, "Damage Detection in Offshore Structures by the Random Decrement Technique," ASME Journal of Energy Resources Technology, Vol. 106, pp. 38-42.

Yun, C.-B., and Hong, K.-S., 1992, "Damage assessment of structures by inverse modal perturbation method," Proc. 4th East Asia-Pacific Conf. Structural Engineering and Construction, Seoul, Korea.

Yun, C.-B., Lee, H.-J., and Lee, C.-G., 1997, "Sequential prediction error method for structural identification," Journal of Engineering Mechanics, ASCE, Vol. 123, No. 2, pp. 115-122.

Yun, C.-B., and Shinozuka, M., 1980, "Identification of Nonlinear Structural Dynamic Systems," Journal of Structural Mechanics, Vol. 1, pp. 187-203. 